\title{
XII. Zeitzeugnis und Zeitgeschichte
}

Rudolf Morsey, in diesem Zusammenhang sowohl Zeitzeuge wie Akteur ${ }^{1}$, nennt Johannes Schauff den Initiator der später mit der „Kommission für Zeitgeschichte " institutionalisierten Erforschung der jüngsten Vergangenheit, insbesondere der des Katholizismus; er sieht in Schauffs Engagement eine "quasi geistige Entwicklungshilfe" 2 . Die Notwendigkeit einer wissenschaftlichen Aufarbeitung der Rolle der demokratischen Kräfte und vor allem des Zentrums beim Niedergang der Weimarer Republik war Schauff frühzeitig bewußt geworden ${ }^{3}-$ schon $^{2}$ seine Aufzeichnungen an Bord der "Oceania“ aus dem Jahre 1934 sind hierfür ein beredtes Zeugnis ${ }^{4}$.

Wesentlich später, in Zusammenhang mit der Vernichtung des Nachlasses von Ludwig Kaas, dessen Politik er in den "Oceania“-Aufzeichnungen vernichtend kritisiert hatte und der dann selbst in großherziger Weise bemüht war, der nach Rom emigrierten Familie Schauff zu helfen ${ }^{5}$, geht Johannes Schauff auf seine Zielsetzungen in diesem Zusammenhang noch einmal ausführlich ein. In dieser Denkschrift von 1961 heißt es, daß Kaas in den letzten Jahren seines Lebens in „vielen freundschaftlichen Gesprächen über das ,Ende der Weimarer Zeit “ " bedauert habe, „daß er seine Akten aus jener Zeit im Auftrag von Papst Pius XII., als man

1 Morsey (geb. 1927) war 1966-1970 Ordinarius für Neuere und Neueste Geschichte an der Universität Würzburg, 1970 bis zu seiner Emeritierung 1996 o. Prof. für Neuere Geschichte an der Hochschule für Verwaltungswissenschaften in Speyer; 1968-1998 Vorsitzender der Kommission für Geschichte des Parlamentarismus und der politischen Parteien in Bonn.

2 Morsey, Ein ungewöhnliches deutsches Schicksal: Johannes Schauff, S. $104 \mathrm{f}$. Nachfolgende Ausführungen basieren auf dem umfangreichen Briefwechsel Schauffs vor allem mit Morsey (IfZ, NL Schauff, Bd. 5 u. Bd. 35), der auch in: Morsey, Gründung und Gründer, S. 453-485 Eingang gefunden hat. Schauff teilte dem Verfasser am 12.7. 1988 in einer Notiz mit: „An Professor Morsey schicke ich die Unterlagen zur Kommission für Zeitgeschichte und ihrer Gründung." Morseys Darstellung unter Berücksichtigung auch der vorangegangenen Literatur dokumentiert den Forschungsstand zur Gründungsgeschichte der Kommission.

3 Einer der politischen Weggefährten, mit denen Schauff bereits früh die Notwendigkeit einer wissenschaftlichen Aufarbeitung dieser Vorgänge erörterte, war Karl Thieme (siehe oben, S. 38 sowie Anm. 72), mit dem Schauff auch während der Exiljahre in Verbindung stand. Von Thieme stammt ein Projektentwurf über das „Erliegen der Deutschen Zentrumspartei im Kampf gegen Hitler ${ }^{\alpha}$ vom 18.1. 1949. Als Betreuer und Autor wurde Georg Smolka vorgesehen, der zu dieser Zeit mit einer Gastprofessur an der Staatlichen Akademie für Verwaltungswissenschaften in Speyer betraut war. Smolka war vor 1933 Mitarbeiter der Zeitschrift Der Ost-Siedler und von daher auch Schauff unmittelbar bekannt (vgl. die biographische Vorbemerkung von Morsey, in: Smolka, Die Auswanderung, S. 1-7). Dem Projekt sollte ein „Herausgeberkomitee“ zur Seite gestellt werden, für das die Professoren Clemens Bauer (Freiburg), Wilhelm Neuß (Kirchenhistoriker in Bonn) sowie Franz Schnabel (München) vorgesehen wurden (IfZ, NL Schauff, Bd. 8).

4 Siehe oben, S. $54 \mathrm{ff}$.

5 Karin Schauff, Ludwig Kaas; die nachfolgend zitierte Denkschrift von Schauff „Zum politischen Nachlaß von Mons. Kaas“ (Januar 1961), ebenda, S. $27 \mathrm{f}$. 
im Verlauf des Krieges mit einer Besetzung des Vatikans durch die SS rechnen mußte, vernichtet hat".

Kaas habe vorausgesehen, daß die historischen Vorgänge um die Entlassung Brünings, um dessen Vatikanbesuch, um das Ermächtigungsgesetz und um das Reichskonkordat später heftig umstritten sein würden. Er, Schauff, sei mit Kaas bereits 1949 in der Frage einig gewesen, „daß diese Zeit, vor allem das Verhältnis von Kirche und Staat betreffend, von Historikern erforscht werden müßte“. Dazu sollten einschlägiges Quellenmaterial gesammelt sowie Zeugen befragt werden, um rechtzeitig Vorsorge für eine spätere objektive Geschichtsforschung zu treffen.

Er, Schauff, habe diese Gedanken aufgenommen und mehrere Verhandlungen u.a. mit Heinrich Brüning, Prälat Wilhelm Böhler, Heinrich Vockel, Heinrich Krone, Prälat Hermann Joseph Schmitt, Pater Leiber und Andreas Hermes geführt, „um eine solche zentrale Archivierungs- und Bearbeitungsstelle, besetzt mit Fachhistorikern, schaffen zu lassen. Prälat Kaas wirkte sogar bei der Vorbereitung eines entsprechenden Fragebogens zu den am meisten umstrittenen Fragen mit, $z u$ dessen Beantwortung er sich ebenso wie zu einer mündlichen Unterredung mit kompetenten Historikern verpflichtete. Meine Bemühungen waren jedoch zunächst ergebnislos, vor allem da H.[einrich] Brüning sich ausdrücklich versagte, und auch andere die Situation noch nicht reif für die Klärung hielten. Das negative Ergebnis wurde von Kaas sehr bedauert."

Keineswegs entmutigt, benützte Schauff nach dem Tode von Kaas die anvisierten „Verhandlungen“ zur Sammlung von Quellen zum Kontakt mit interessierten Zeithistorikern. Auf seine Anregung hin unternahm Heinrich Krone am 22. September 1952 einen Vorstoß, die Geschichte der beiden Kirchen im Dritten Reich wissenschaftlich erforschen zu lassen ${ }^{6}$. Krone verhandelte mit dem Bundesministerium des Innern wegen der Gewährung entsprechender Stipendien, um den Kampf beider Kirchen im Dritten Reich durch zwei jüngere Historiker untersuchen zu lassen. Für die Bearbeitung des katholischen Teils wurde ursprünglich Georg Smolka vorgesehen, dem Karl Thieme bereits die gleiche Aufgabe hatte zuweisen wollen; 1954 übernahm dann der Berliner Kirchenhistoriker Bernhard Stasiewski den Auftrag einer entsprechenden Quellensammlung7.

Schauffs Bemühungen um eine historische Aufarbeitung des politischen Katholizismus in der Weimarer Republik und der NS-Zeit wurden in diesem Zeitraum vor allem von zwei Persönlichkeiten unterstützt: zum einen der junge Historiker Rudolf Morsey, auf der anderen Seite der ebenfalls noch junge Direktor der Katholischen Akademie in Bayern, Karl Forster. Morsey, zu dieser Zeit Mitarbeiter der „Kommission für Geschichte des Parlamentarismus und der politischen Parteien", hatte Schauff 1958 im Haus des Kirchenhistorikers und früheren Zentrumsabgeordneten Georg Schreiber in Münster kennengelernt. Über Schreiber

6 Vgl. hierzu auch Repgen, Kommission für Zeitgeschichte, S. 9-17, hier S. 10 f.

7 Krone, Tagebücher, Bd. I, S. 136 f. (Eintrag 14. 5. 1954). Die von Stasiewski (1905-1995) bearbeiteten Bände I-III der Edition der „Akten deutscher Bischöfe über die Lage der Kirche" für die Jahre 1933-1936 erschienen 1968-1979 in den Veröffentlichungen der Kommission für Zeitgeschichte. 
stand Morsey auch in Verbindung mit dem „Böhler-Kreis" 8 und machte in diesem Zusammenhang auch die Bekanntschaft von Heinrich Krone?.

Auch mit dem ehemaligen Fraktionskollegen Schreiber, der wie er in Opposition zum NS-Regime gestanden hatte und verfolgt worden war ${ }^{10}$, traf Schauff sich in dem gemeinsamen Interesse an zeitgeschichtlicher Forschung. Durch den sogenannten Konkordatsstreit - den Konkordatsprozeß vor dem Bundesverfassungsgericht 1955/5611 - wurde Schauff weiter in seiner Motivation bestärkt, einer politischen Legendenbildung über das Verhältnis von Staat und Katholischer Kirche entgegenzuwirken.

Um Vorwürfen zu begegnen, die Kirche habe bestimmte Dinge zu verbergen oder erschwere deren historische Erforschung - dies galt allem für die Rolle von Heinrich Brüning und Ludwig Kaas -, plante er 1959 einen Sammelband mit Reden und Aufsätzen von Kaas, da mit den erwarteten Memoiren von Heinrich Brüning nicht $\mathrm{zu}$ rechnen sei ${ }^{12}$. Für eine Vorrede zu dieser Edition wollte er sowohl Konrad Adenauer wie Kardinal Montini und die amerikanische Botschafterin in Rom, Claire Boothe Luce, gewinnen ${ }^{13}$. Das Projekt konnte nicht realisiert werden - statt dessen gewann die Idee an Boden, Zeitzeugen und Historiker zusammenzuspannen. Dafür konnte Schauff die Unterstützung von Karl Forster gewinnen ${ }^{14}$, der zudem hohes Interesse daran besaß, einschlägiges Quellenmaterial in der Katholischen Akademie zu archivieren. Von Rudolf Morsey ermu-

8 Im Kreis um den Leiter des Katholischen Büros in Bonn, Prälat Wilhelm Böhler (18911958), war eine Denkschrift zur Begründung eines Instituts zur Erforschung der Geschichte des katholischen Deutschlands im 18. und 19. Jahrhundert verfaßt worden. Vgl. Repgen, Kommission für Zeitgeschichte, S. 10f.; Morsey, Gründung und Gründer, S. $455 \mathrm{f}$.

9 Morsey, Gründung und Gründer, S. 459.

10 Vgl. Morsey, Georg Schreiber, S. 269-284; M.d.R., Die Reichstagsabgeordneten der Weimarer Republik und die Zeit des Nationalsozialismus, S. 1397f. (mit weiterführender Literatur).

11 Das Reichskonkordat von 1933 galt nach 1945 für das Verhältnis der Bundesrepublik Deutschland zum Heiligen Stuhl weiter. Da die Länder den "Schulartikel“ jedoch nicht anerkannten, kam es in dieser Frage zum „Konkordatsstreit“; 1957 entschied das Bundesverfassungsgericht, daß die Länder dem Reichskonkordat in dieser Frage nicht zu folgen hätten.

12 Johannes Schauff, Vorschlag für eine Klausurtagung von Historikern und Politikern zum Thema „Kirche und Staat am Ausgang der Weimarer Zeit“, Ms. (IfZ, NL Schauff, Bd. 24), S. 1.

13 Zit. nach, Gründung und Gründer, S. 460. Dort auch der Gliederungsentwurf: „Deutsche Zeit“ (mit vier Texten), „Römische Zeit“ (mit drei Texten), ergänzt um insgesamt sechs "Anlagen“, darunter das "Thomasbild“ am Portal des Petersdoms, das „ihn [Kaas] darstellt“. Eine Sammlung der Reden und Aufsätze von Kaas unter dem Titel „Zwischen Rhein und Reich, Kirche und Politik" von Rudolf Morsey ist nicht veröffentlicht.

14 Schauff informierte am 2. 2. 1960 Heinrich Krone, daß er mit Forster den Plan einer „Historiker-Tagung über die Zeit von $1933^{\text {“ }}$ erörtert habe (ACDP, NL Heinrich Krone, I-028-014/1); am 23. 3. 1960 schrieb er an Morsey, daß er bei einem kürzlichen Aufenthalt in Bonn nicht die Zeit gefunden habe, über eine „Katholische Akademietagung über die Zeit zwischen Brüning und Konkordat" zu sprechen; Morsey wies in seiner Antwort (29. 3. 1960) darauf hin, daß man kompetente und mutige Referenten gewinnen müsse. Zit. nach Morsey, Gründung und Gründer, S. 461. 
tigt, bot Schauff ihm Materialien aus dem Kaas-Nachlaß zur Veröffentlichung an, die er erst einige Monate zuvor in Sterzing entdeckt hatte. Er glaubte, mit diesen Dokumenten belegen zu können, daß es keinen „Zusammenhang zwischen der Zustimmung der Zentrumsfraktion zum Ermächtigungsgesetz und dem Konkordatsabschluß " gegeben habe ${ }^{15}$. Die Morsey von Schauff „zu treuen Händen und vertraulich" am 11. Juni 1960 übergebenen Unterlagen wurden in den Stimmen der Zeit veröffentlicht; deren Herausgeber Oskar Simmel SJ konnte für eine Veröffentlichung der tagebuchartigen Aufzeichnungen von Kaas gewonnen werden ${ }^{16}$.

Bereits Juli 1960 war Morseys Beitrag über das Ende der Zentrumspartei in dem historiographisch nach wie vor unverzichtbaren Sammelwerk über das „Ende der Parteien 1933“ erschienen ${ }^{17}$. Auch laut Karin Schauff löste er eine „Flut von Stellungnahmen “ aus, „nicht zuletzt von früheren Zentrumspolitikern und Abgeordneten, die aus ihrer Erkenntnis und Erfahrung manche Zusammenhänge anders beurteilten. Das galt vor allem für die Rolle von Ludwig Kaas." 18

Johannes Schauff selbst sah Morseys Untersuchung als Ergänzung und Fortführung der bisher von Prälat Wilhelm Böhler geförderten Forschungsarbeiten, die nunmehr „weitere Quellen zur Haltung des Zentrums sowie der deutschen Bischöfe in den Jahren 1930-1933“ erschließe. Die Studie führe außerdem frühere Arbeiten zur Kirchengeschichte der Weimarer Zeit fort, „die in ,Hochland“ (Karl Buchheim, Clemens Bauer), im ,Historischen Jahrbuch“ der Görresgesellschaft (Prälat Georg Schreiber, Weihbischof Anton Scharnagl), in den ,Politischen Studien' (Prälat Böhler), in der ,Zeitschrift der Savigny-Stiftung für Rechtsgeschichte' (Rudolf Morsey) und im ,Rheinischen Merkur' (Adolf Süsterhenn) erschienen sind“.

Die von Rudolf Morsey publizierten Kaas-Materialien hatten nach Schauffs Meinung überdies in einer Hinsicht für endgültige Klarstellung gesorgt: Damit sei „der 1956 im Zusammenhang mit dem Konkordatsprozeß vor dem Bundesverfassungsgericht sowohl von seiten der Politischen Wissenschaftler (Karl Dietrich Bracher) als auch der SPD (Adolf Arndt, Friedrich Stampfer) erhobene Vorwurf widerlegt worden, Kaas habe als Vorsitzender des Zentrums die Zustimmung seiner Fraktion zum Ermächtigungsgesetz ... mit einer Zusage Hitlers erkauft, ein Reichskonkordat abzuschließen“19.

15 Schauff, Zum politischen Nachlaß von Monsignore Kaas (vgl. Anm. 5).

16 Es handelte sich neben diesen tagebuchartigen Aufzeichnungen von Kaas aus Rom für die Zeit vom 7.-20.4. 1933 um eine Niederschrift des Vorsitzenden der Reichstagsfraktion des Zentrums, Ludwig Perlitius, über die Verhandlungen von Kaas und Perlitius am Vormittag des 31. Januar 1933 mit Hitler und Reichsinnenminister Frick (wegen einer eventuellen Beteiligung an der neugebildeten Regierung bzw. der Möglichkeit ihrer Tolerierung, siehe oben, S. 55) sowie um Kaas' Briefwechsel mit Papen zum Reichskonkordat für die Zeit vom 20. 4.-11. 7. 1933. Veröffentlichung in Stimmen der Zeit 166 und 167 (1960/61).

17 Morsey, Die Deutsche Zentrumspartei, S. 282-453.

18 Karin Schauff, Ludwig Kaas, S. 28.

19 Schauff, Vorschlag für eine Klausurtagung (IfZ, NL Schauff, Bd. 24, S. 3 f.). 
Bis zur Institutionalisierung der derart eingeleiteten, doch bisher weitgehend individualisierten Forschungsarbeit war es jedoch noch ein langer Weg. Die Veröffentlichung der Kaas-Materialien sowie Morseys Studie fanden keineswegs die einhellige Zustimmung der früheren politischen Akteure, aber auch nicht in vatikanischen Kreisen ${ }^{20}$. Hinzu kam das latente Mißtrauen mancher Zeitzeugen gegenüber jüngeren und ihnen persönlich kaum bekannten Historikern.

Schauff bildete hier eine rühmliche Ausnahme. Seine Zusammenarbeit mit Morsey, von dem er immer wieder „Argumentationshilfe“ erbat, hatte - neben aller von Schauff bei Morsey geschätzten fachlichen Kompetenz - mit seiner prinzipiellen Bereitschaft zu tun, der Jugend und dem wissenschaftlichen Nachwuchs Vertrauen entgegenzubringen und sie zugleich und rechtzeitig in Verantwortung einzubinden. Dies entsprach seiner grundsätzlichen Haltung, die aufgrund der Erfahrungen mit der großen Familie und unter den anfangs unsäglich schwierigen Bedingungen des Exils eher gewachsen war. In einer solchen von Schauff erbetenen Argumentationshilfe etwa übermittelte ihm Morsey am 11. Oktober 1960 eine Aufzeichnung "Zur Veröffentlichung zeitgeschichtlicher Quellen“, die die Erschließung des vorhandenen einschlägigen Quellenmaterials sowie die Notwendigkeit betraf, das Zeitzeugnis ehemaliger Zentrumspolitiker einzuholen ${ }^{21}$.

Im weiteren Verlauf vermochte das Tandem Schauff-Morsey die Institutionalisierung kirchlicher Zeitgeschichte voranzubringen. Nach „weiterer Abklärung mit Schauff und Forster über die Möglichkeit einer Klausurtagung" am 28. Oktober 1960 schlug Morsey für eine Tagung das Thema „Der deutsche Katholizismus und die Staatskrise 1930-1933“ vor. Veranstalter dieser Tagung sollte die Katholische Akademie in Bayern in Verbindung mit dem Katholischen Büro in Bonn sein, Tagungsort Würzburg. Als Termin wurde Februar/März 1961 vorgeschlagen und als potentielle Referenten August H. Berning22, Robert Leiber, Ernst Deuerlein ${ }^{23}$ sowie Rudolf Morsey ${ }^{24}$.

Schauff versuchte seinerseits, frühere Fraktionskollegen für eine solche Tagung zu gewinnen und erbat weitere "Argumentationshilfe“, um skeptische Zeitzeugen zu gewinnen; sie fand ihren Niederschlag in einer Neufassung des Morseyschen Tagungsentwurfs ${ }^{25}$. Darin wurde noch einmal festgehalten, daß die Klausurtagung einen Beitrag leisten solle „zur Klärung von politischen Zusammenhängen

20 So äußerte sich z.B. Heinrich Brüning, dem Heinrich Krone die Dokumentation geschickt hatte, „entsetzt darüber, daß gerade die Stimmen der Zeit die Vorgänge um Kaas enthüllen" (Brüning an Krone, 16.11. 1960, ACDP I-028-014/1). Diese Kritik war Morsey von Schauff am 22. 9. 1960 übermittelt worden (Morsey, Gründung und Gründer, S. 464). Kritische Äußerungen kamen auch von Pater Leiber (so in den Stimmen der Zeit, 165, 1960/61); Morsey, Gründung und Gründer, S. 463.

21 Morsey, Gründung und Gründer, S. 465.

22 Berning (1895-1979) war vor 1933 Kulturreferent im Berliner Generalsekretariat des Zentrums.

23 Deuerlein, Neu- und Zeithistoriker, war ab 1964 o. Prof. an der Philosophisch-Theologischen Hochschule in Dillingen.

24 Morsey, Gründung und Gründer, S. $465 \mathrm{f}$.

25 Der Entwurf ist datiert vom 1.12. 1960 (Vorschlag für eine Klausurtagung, IfZ, NL Schauff, Bd. 24). 
einer Zeit, über die noch allzu gern der Schleier des Vergessens und Verschweigens gebreitet wird“; außerdem solle verdeutlicht werden, „daß in der jetzt beginnenden zweiten Phase der Auseinandersetzung über diese Zeit eine Konfrontierung mit den Vorgängen der letzten 30 Jahre auch für das zukünftige deutsche Schicksal eher förderlich als belastend ist“.

Am 19. September 1960 schlug Morsey Schauff und Forster vier Referatsthemen zum Tagungsthema „Katholizismus und Staatskrise 1930-1933“ vor und nannte potentielle Referenten ${ }^{26}$. Über das Projekt einer „geschlossenen Tagung“ sprach Schauff am 2. Januar 1961 mit dem befreundeten Bruno Wüstenberg vom Vatikanischen Staatssekretariat, worauf jener Morsey über negative Äußerungen aus dem Kreis älterer Mitglieder des Episkopats informierte. Schauff empfahl, Kardinal Bea auf dem laufenden zu halten und das „Memorandum“, das er auch Bischof Hengsbach zuleiten wollte, vor allem unter Berücksichtigung der Kritik von Leiber an Morseys Thesen über den Untergang des Zentrums etwas zu modifizieren; diese Kritik betreffe auch die kritische Einschätzung der Rolle von Kaas im Jahre 193327. Am 20. Januar 1961 lieferte Morsey eine zweite, erweiterte und entsprechend überarbeitete Fassung der Denkschrift, die Schauff sogleich weiterleitete ${ }^{28}$. Bei einem Treffen am 2. März 1961, an dem Forster, Schauff, Karl Buchheim, Johannes Hirschmann und Morsey teilnahmen, wurden die letzten Details der geplanten Tagung besprochen; als Termin einigte man sich wenig später auf den 8. und 9. Mai 1961.

In der von Karl Forster formulierten Einladung zu einer „geschlossenen Veranstaltung" über das Thema „Die Katholiken und das Schicksal der Weimarer Republik" hieß es mit Blick auf die eigentlichen Adressaten, die früheren politischen Akteure: „Anlaß zur Planung sind verschiedene Veröffentlichungen, die sich in letzter Zeit mit der politischen Rolle des deutschen Katholizismus am Ende der Weimarer Republik befaßt haben. Historische Arbeiten widersprechen sich teilweise in der Darstellung wichtiger Vorgänge. Kommunistische Äußerungen benützen bestimmte Aktenstücke als Grundlage für hemmungslose Propaganda ${ }^{29}$. Linkskatholische Thesen zur politischen Verantwortung des katholischen Christen berufen sich auf Vorgänge, die durch ihre isolierte Hervorhebung zu Fehlurteilen führen." Der wichtigste Grund für das Vorhaben der Akademie

26 Karl Buchheim, August H. Berning, Ernst Deuerlein, Robert Leiber SJ, Johannes Hirschmann SJ und Rudolf Morsey (Morsey, Gründung und Gründer, S. 467).

27 Ebenda, S. $467 \mathrm{f}$.

28 Adressaten waren Max Braubach, Wilhelm Fonk, Paul Franken, Andreas Hermes, Johannes Hirschmann SJ, Robert Leiber SJ, Hermann Joseph Schmitt, Karl Schwend, Georg Smolka, Christine Teusch und Heinrich Vockel (ebenda, S. 468).

29 Zur Genesis dieser Aussagen gehören Schauffs einschlägige Erkenntnisse: „Auch um die Persönlichkeit von Papst Pius XII. und seine Haltung gegenüber Deutschland während und nach seiner Tätigkeit als Nuntius in München und Berlin (1917-1929) und als Kardinalstaatssekretär haben sich vielfach Legenden gerankt. Sie steigerten sich bis zu scharfen Angriffen der sowjetzonalen Geschichtswissenschaft ..., wobei die in Potsdam (ehemaliges Reichsarchiv) lagernden amtlichen deutschen Akten als Materialgrundlage benutzt werden. Andere kommunistische Autoren haben massive Beschuldigungen gegen den ,Klerikalismus' und die vatikanische Politik in Deutschland erhoben ..." (Vorschlag für eine Klausurtagung, IfZ, NL Schauff, Bd. 24, S. 1 f.). 
sei daher „das Bestreben, das politische Wirken des deutschen Katholizismus in den Jahren wichtiger Entscheidungen in das Licht der vollen geschichtlichen Wahrheit zu rücken... Da die Aktenlage mit der Annäherung an die Zeit der nationalsozialistischen Herrschaft immer lückenhafter und damit unzuverlässiger wird, kommt dem Erfahrungsaustausch zwischen katholischen Historikern und den damals politisch tätigen Persönlichkeiten eine besondere Bedeutung zu. “ 30

Vorbehalte von seiten der Zeitzeugen, die die Historiker als allzu aktengläubig beurteilten, waren damit freilich noch nicht ausgeräumt. Sowohl Heinrich Krone, der ursprünglich zu den ersten Förderern des Projekts gehört hatte, wie auch Andreas Hermes und Christine Teusch, beide ebenfalls ehemalige Reichstagsabgeordnete des Zentrums, erneuerten ihre Kritik, so daß Schauff sich erneut um Schadensbegrenzung bemühen mußte. Am 17. April 1961 wandte sich Schauff telegraphisch an Christine Teusch und erklärte seine Bereitschaft zu einer zusätzlichen Besprechung im kleinen Kreise, hielt jedoch ausdrücklich an dem festgelegten Tagungstermin fest. Krone gegenüber präzisierte er das Vorhaben als ein Projekt von der Art eines „Katholischen Instituts für Zeitgeschichte“ und wies in diesem Zusammenhang explizit auf die wohlwollende Haltung Kardinal Beas hin ${ }^{31}$. Gegenüber Christine Teusch nahm Schauff später den Vorschlag eines vorgezogenen Treffens im kleineren Kreis wieder zurück, um „möglichen Mißverständnissen“ vorzubeugen ${ }^{32}$.

Schauff betonte in diesem Zusammenhang, ein ähnlicher Versuch sei bereits 1949 gescheitert; er bemühe sich deshalb um eine detailliertere Erklärung des neuen Projekts, bei dem es darauf ankomme, „daß den jungen Historikern die Komplexheit der Probleme der damaligen Zeit klar wird und als Ergebnis eine Art ,Katholisches Institut für Zeitgeschichte' eingerichtet wird, das Nachlässe, Erinnerungen und Dokumente sammelt und unter sachkundiger und verantwortungsvoller Leitung - unter Diskretion - bearbeitet“. Eine zusätzliche Argumentationshilfe war sicherlich die 1961 in den Vierteljabrsheften für Zeitgeschichte veröffentlichte Dokumentation „Hitlers Verhandlungen mit der Zentrumsführung am 31. Januar 1933“, der das Protokoll des ehemaligen Fraktionsvorsitzenden des Zentrums im Reichstag, Ludwig Perlitius, zugrunde liegt; Schauff hatte dieses Protokoll Morsey zugänglich gemacht ${ }^{33}$.

30 Die Einladung informierte ebenfalls über den vorgesehenen Tagesablauf und die Referate: Karl Buchheim, „Die Stellung der deutschen Katholiken zum Weimarer Staat“; Johannes Hirschmann, „Der innerkirchliche Strukturwandel der zwanziger Jahre als Hintergrund des politischen Handelns“; Konrad Repgen, „Die Staatskrise ab 1930 und die deutschen Katholiken“; Rudolf Morsey, „Die Kirche und die Machtübernahme durch den Nationalsozialismus“ (zit. nach Morsey, Gründung und Gründer, S. 470).

31 Schauff war in Rom mit den Kardinälen Muench und Bea zusammengetroffen (Schauff an Krone, 17. 4. 1961, ACDP, I-028-014/1); an Morsey die Nachricht vom gleichen Tage, daß Kardinal Bea sehr positiv über die Tagung gesprochen habe (Morsey, Gründung und Gründer, S. 472).

32 Schreiben Schauff an Christine Teusch, das erst am 1. 5. 1961 von München abgeschickt wurde (ACDP, I-028-014/1).

33 Morsey, Hitlers Verhandlungen; im gleichen Heft der VfZ die Dokumentation von Josef Becker, Zentrum. 
Danach waren interne Widerstände weitgehend ausgeräumt, und am 8. und 9. Mai 1961 fand im Burkardus-Haus in Würzburg die vorbereitete Klausurtagung statt. An ihr nahmen neben elf ehemaligen Zentrumspolitikern (Johannes Schauff, Josef Beyerle, Oskar Farny, Wilhelm Fonk, Heinrich Krone, Joseph Joos, Joseph Kannengießer, Hermann Joseph Schmitt, Leo Schwering, Helene Weber und August Wegmann) und anderen Zeitzeugen (unter ihnen Paul Franken, Prälat Bernhard Hanssler und Robert Leiber SJ) die Historiker Hans und Karl Buchheim, Ernst Deuerlein, Rudolf Morsey, Konrad Repgen, Albert Schwarz, Georg Smolka und Bernhard Stasiewski sowie der Würzburger Jurist Paul Mikat teil; anwesend waren auch mehrere Publizisten (August H. Berning, Klaus Dohrn, Heinrich Scharp und Karl Schwend). Auf der Tagung referierten Karl Buchheim, Johannes Hirschmann SJ, Konrad Repgen und Rudolf Morsey34. Von seiten der ehemaligen Akteure wurde allerdings erneut der Vorwurf laut, daß sich die Historiker zu sehr auf schriftliche Überlieferungen verließen: Alle früheren Zentrumsabgeordneten betonten, daß es keinen Zusammenhang zwischen ihrer $\mathrm{Zu}$ stimmung zum Ermächtigungsgesetz und dem Abschluß des Reichskonkordats gegeben habe.

Zur weiteren Institutionalisierung der Katholizismus-Forschung wurde von den Teilnehmern der Würzburger Tagung ein Fünfergremium eingesetzt, bestehend aus Hans Buchheim, Forster, Hanssler, Krone und Morsey, das, wie in der Pressemeldung zur Tagung mitgeteilt wurde, die Gründung eines „Katholischen Instituts für Zeitgeschichte" vorantreiben solle ${ }^{35}$. Zumindest der Name wurde in deutlicher Analogie zum Institut für Zeitgeschichte in München gewählt, zu dem in erster Linie über Vater und Sohn Buchheim Beziehungen bestanden ${ }^{36}$. Schauff sollte diese Verbindung, wie noch zu zeigen sein wird, später wissenschaftlich durchaus nutzen. Über den Fortgang bzw. die Konkretisierung der weiteren $\mathrm{Be}$ mühungen hielt Morsey Schauff auf dem laufendem, auch als dieser sich zu einem turnusmäßigen Aufenthalt in Brasilien befand ${ }^{37}$. Inzwischen war abzusehen, daß eine Kommission für Zeitgeschichte bei der Katholischen Akademie in Bayern

34 Es gab folgende Referatthemen: Karl Buchheim, „Die Katholiken und die Republik“; Johannes Hirschmann, „Der innere katholische Strukturwandel der zwanziger Jahre als Hintergrund des politischen Handelns“; Konrad Repgen, „Die Staatskrise ab 1930 und die deutschen Katholiken“, sowie Rudolf Morsey, „Der Katholizismus und die Machtübernahme durch den Nationalsozialismus".

35 Morsey, Gründung und Gründer, S. 474.

36 Karl Buchheim (1889-1972) gehörte zur Gründergeneration des Instituts für Zeitgeschichte, dem er bis 1952 angehörte (vgl. Buchheim, Lebensgeschichte, S. 253 ff.); der Sohn Hans Buchheim war 1950-1966 Wissenschaftlicher Mitarbeiter des Instituts.

37 Forster wollte für die Anfang 1962 geplante Forschungsstelle den Münchner Privatdozenten Dieter Albrecht gewinnen; in einem Gespräch der nach dem Würzburger Treffen gebildeten "Fünferkommission" am 27. 10. 1961 bei Heinrich Krone in Bonn wurde vereinbart, ein "Kuratorium“ zu bestellen, das seinerseits ein „historisch-wissenschaftliches Gremium" berufen sollte. Diese Informationen übermittelte Morsey am 27.10. 1961 Schauff, der daraufhin Krone für dessen „Bemühungen“ dankte (8.11. 1961, ACDP, I-028-014/1). An Morsey schrieb Schauff am gleichen Tag: „Ich bin froh, daß wir einen Schritt weiter sind und Dr. Krone das Versprechen mir gegenüber wahrgemacht hat."(Morsey, Gründung und Gründung, S. 476f.). 
eingerichtet werden würde. In einem Gespräch der „Fünferkommission“ am 24. Januar 1962 wurden schließlich die anstehenden organisatorischen Fragen wie die Gliederung der neuen Kommission erörtert. Danach sollten in einen „Verwaltungsrat" sowohl ehemalige Politiker des Zentrums und der Bayerischen Volkspartei wie Vertreter des öffentlichen katholischen Lebens berufen werden: Man dachte an den Leiter des Katholischen Büros in Bonn, Prälat Wilhelm Wissing, den Geistlichen Direktor beim Zentralkomitee der Deutschen Katholiken, Prälat Bernhard Hanssler, und den Präsidenten der Görres-Gesellschaft, Hans Peters. Dem zu bildenden "Wissenschaftlichen Beirat" sollten neben Wissenschaftlern wie Hans und Karl Buchheim, Deuerlein, Mikat, Morsey, Repgen, Roegele, Albert Schwarz, Smolka und Stasiewski, die bereits an der Würzburger Tagung teilgenommen hatten, noch weitere Historiker angehören: Vorgeschlagen wurden Dieter Albrecht, Max Braubach, Hubert Jedin, Bernhard Zittel sowie der Sozialwissenschaftler Gustav Gundlach.

Am 13. April 1962 fand in Bonn ein weiteres Treffen statt, an dem Schauff, Forster, Morsey, Krone und Fonk teilnahmen. Hierbei wurden die später offiziellen „Rahmenbedingungen über die Errichtung einer Kommission für Zeitgeschichte bei der Katholischen Akademie in Bayern" diskutiert und präzisiert. Am 5. Mai 1962 konnte Schauff Dieter Sattler berichten, das „Katholische Institut für Zeitgeschichte" nehme nunmehr „feste Formen" an ${ }^{38}$.

Am 17. September 1962 konstituierte sich in der Katholischen Akademie zunächst der Wissenschaftliche Vorstand und dann das Kuratorium der „Kommission für Zeitgeschichte“, nachdem die deutsche Bischofskonferenz - nach vorangehender Intervention und Information von seiten Forsters - 35000 DM als Unterstützung bewilligt hatte. Schauff hatte über die bevorstehende Gründung der Kommission Theodor Heuss informiert, der daraufhin die „Untersuchung über die Haltung des politischen Katholizismus in den Krisenjahren" begrüßte, nicht zuletzt um der Gefahr zu begegnen, daß sich ein „Legendenmantel" bilde 39 .

Dem ersten Kuratorium, zu dessen Vorsitzenden Wilhelm Fonk gewählt wurde, gehörten an: Hans Berger (Botschafter in Kopenhagen), Karl Forster, der ehemalige Zentrums-MdR Wilhelm Fonk, Joseph Ernst Fürst Fugger von Glött

38 IfZ, NL Dieter Sattler, Bd. 90.

39 „Es ist wohl gut, wenn diese Dinge einmal dokumentarisch und aus dem Urteil einzelner Menschen festgehalten werden, denn die Gefahr besteht, daß sich ein Legendenmantel bildet. Vielleicht wird Heinrich Brüning durch die Gründung der Gruppe veranlaßt, seine Reserve zu verlassen. Ich freue mich, daß mein Freund Gottfried Treviranus bei der Veranstaltung mitwirken wird. Er unterhält, so weit ich sehe, fast als Einziger intensivere $\mathrm{Be}-$ ziehungen zu Brüning. Es müßte nach meinem Gefühl auch Stegerwalds Figur verdeutlicht werden und wenn dies möglich ist, die Beziehung zwischen Adenauer und Brüning (hier als jüngstes Forschungsergebnis: Morsey, Brüning und Adenauer). Vor vielen Jahren hat einmal ein persönlicher und politischer Freund von Brüning mir erzählt, dieser habe sich gegen ein Reichskonkordat mit der römischen Kurie gewehrt, weil er den Standpunkt vertreten habe, derlei müsse von einem protestantischen Reichskanzler, wenn überhaupt, abgeschlossen werden. Das kann schon ein Stück von der Legendenbildung sein, von der ich vorhin sprach." (Heuss an Schauff, 29. 8. 1962, IfZ, NL Schauff, Bd. 34); vgl. auch Morsey, Gründung und Gründer, S. $479 \mathrm{ff}$. 
(CSU-MdB) ${ }^{40}$, Karl Theodor Freiherr von und zu Guttenberg (CSU-MdB), Prälat Bernhard Hanssler, Prälat Wilhelm Wissing, Hans Peters, Johannes Schauff und der frühere bayerische Ministerpräsident und Bundesminister Fritz Schäffer. Mitglieder des Wissenschaftlichen Vorstands waren Dieter Albrecht, Clemens Bauer, Karl Bosl, Hans Buchheim, Karl Buchheim, Ernst Deuerlein, Gustav Gundlach SJ, Josef Höfer, Hubert Jedin, Paul Mikat, Rudolf Morsey, Konrad Repgen, Otto B. Roegele, Max Spindler, Bernhard Stasiewski und Bernhard Zittel $^{41}$. Im Anschluß an die Konstituierung der Kommissionsgremien fand vom 17. bis 19. September 1962 die zweite "geschlossene Arbeitstagung" mit dem Thema "Die politische Wirksamkeit der deutschen Katholiken 1928-1934" in München statt ${ }^{42}$.

Mit der konstituierenden Sitzung des Vorstands und des Kuratoriums am 17. September 1962 waren die Bemühungen um die Gründung einer „Kommission für Zeitgeschichte" erfolgreich abgeschlossen worden; auf der Sitzung des Wissenschaftlichen Vorstands am 3. Dezember 1963 wurde Konrad Repgen zum Vorsitzenden und Dieter Albrecht zum geschäftsführenden Mitglied gewählt. Am 28. November konnte Johannes Schauff, nach Morsey „der eigentliche Initiator dieses Projekts“, 1962 seinem Freund Karl Thieme schreiben: „Die zeitgeschichtliche Kommission läßt sich gut an." ${ }^{43}$

Die „Kommission für Zeitgeschichte“ - ihr endgültiger Name wurde auf der Sitzung vom 3. März 1963 festgelegt - war organisatorisch und räumlich bis 1972 mit der Katholischen Akademie in Bayern verbunden. Danach erfolgte die organisatorisch eigenständige Etablierung in Bonn. Das Ergebnis ihrer über dreißigjährigen Forschungs- und Publikationstätigkeit liegt in Form der „Blauen Reihe“ vor - 1998 insgesamt 120 Bände, darunter nicht zuletzt die 1975 auf Initiative von Rudolf Morsey neu aufgelegte Studie Schauffs über „Das Wahlverhalten der deutschen Katholiken im Kaiserreich und in der Weimarer Republik “44.

Schauff, der dem Kuratorium der „Kommission für Zeitgeschichte“ bis 1972 angehörte, blieb eine ihrer wichtigsten Stützen - als Zeitzeuge, der Zugang auch $\mathrm{zu}$ anderen Zeitzeugen und politischen Freunden ermöglichte und auch häufig bei

40 Fugger von Glött hatte dem bayerisch-konservativen Widerstandskreis um den ehemaligen Gesandten Franz Sperr angehört und war nach dem 20. Juli 1944 im gleichen Verfahren wie Helmuth von Moltke und Alfred Delp vom Volksgerichtshof zu drei Jahren Haft verurteilt worden (vgl. Mehringer, Widerstand und Emigration, S. 195 f.).

41 Namensliste nach Morsey, Gründung und Gründung, S. 481.

42 Auf dieser Tagung referierten Rudolf Morsey, „Neue Forschungsergebnisse über die politische Wirksamkeit deutscher Katholiken 1928-1933“; Joseph Joos, „Die geistigen Strömungen und die tatsächlichen Vorgänge in der Entwicklung des Zentrums bis zur Kanzlerschaft Brünings“; Gottfried Reinhold Treviranus, „Anfang und Ende der Kanzlerschaft Brünings“; Robert Leiber, „Die Kirche und der Zusammenbruch der Weimarer Republik“ sowie Hermann Joseph Schmitt, „Vom Ende der Zentrumspartei bis zu den Anfängen des katholischen Widerstands". Ein dritte Tagung wurde von Forster für den Januar 1963 anberaumt: „Das politische Wirken der Katholiken und das Schicksal der Weimarer Republik“ (Morsey, Gründung und Gründer, S. 481 f.).

43 IfZ, NL Karl Thieme, Bd. 70.

44 Hrsg. von Morsey, Mainz 1975. Korrespondenz Schauff-Morsey (5. 3. 1974), IfZ, NL Schauff, Bd. 35. 
der Quellenerschließung und -sammlung hilfreich eingreifen konnte. Sein Engagement war gleichwohl ein kritisches. So monierte er gegenüber Kardinal Döpfner die Loslösung der Kommission von der Katholischen Akademie: Die Zuordnung sei „wesenhaft begründet, weil die Geschichtsforschung und -schreibung des deutschen Katholizismus in der neuesten Zeit nicht von der Akademie als einem Forum der Zeitauseinandersetzung getrennt werden kann. Eine nur fachhistorische, wissenschaftliche Vereinigung rechtfertigt sich $\mathrm{m}$. E. allein nicht. Mit dem Dialog zwischen Politikern und Historikern in Klausurtagungen hat die Kommission begonnen, und diese Besonderheit sollte man nicht unterbrechen. Sie kann m. E. durch eine Distanz zu ,Bonn' eher besser geführt werden. Ferner glaube ich, daß die kommenden Jahrzehnte die deutschen Katholiken nicht aus der Rolle entlassen, nach Nazi- und Adenauerepoche auch unsere ,Zeitperiode kritisch historisch in Zusammenarbeit von Akteuren und Geschichtsschreibern aufarbeiten zu müssen." 45

Im Dialog mit Schauff vermochte Morsey allerdings die Zwänge dieser Entscheidung zu erklären, die u.a. mit gegensätzlichen Konzeptionen des Akademiedirektors Franz Henrich zu tun hätten. Dabei bedankte sich Morsey noch einmal für Schauffs Interesse, mit dem dieser sich im verflossenen Jahrzehnt um die Belange der Kommission gekümmert und darauf geachtet habe, "daß die von Anfang an intendierte Verbindung zwischen den ,Akteuren' und den Historikern nicht gestört wurde. Aus meiner Sicht gibt es keine andere Kommission, in der ein derart erfreuliches und erfolgreiches Zusammenwirken zweier Generationen zu verzeichnen ist, auch wenn dabei (insbesondere in den Anfangsjahren) manche sachliche Kontroversen in den Klausurtagungen ausgetragen wurden." 46 Im Themenrahmen der Kommission für Zeitgeschichte war für Schauff die Emigration unter dem Nationalsozialismus ein wichtiger ergänzender Forschungsgegenstand. Bereits im Exil hatte er mit Arnold Wolfers, mit dem er an der Berliner Hochschule für Politik studiert hatte und der nun in Yale lehrte, Fragen der Migrationsforschung erörtert ${ }^{47}$. Johannes Schauff machte auch das Institut für Zeitgeschichte in München schon frühzeitig auf dieses wichtige Forschungsdesiderat aufmerksam - die ersten Korrespondenzen datieren aus dem Jahre 195848. Bei der Sicherung und Erschließung von Quellen zum politischen Exil in Vorbereitung des Projektes des „Biographischen Handbuchs der deutschsprachigen Emi-

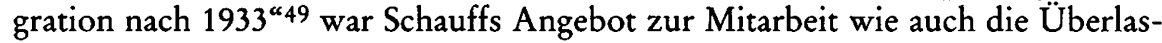
sung seiner Papiere ein wichtiger Beitrag zu dessen Gelingen. Viele solcher Nachlaßgeber hatten nach der Rückkehr aus dem Exil Anschluß an die politischen, administrativen und kulturellen Eliten der Bundesrepublik gefunden oder

45 Schauff an Kardinal Döpfner, Rom, 30. 11. 1972 (IfZ, NL Schauff, Bd. 32).

46 Morsey an Schauff, 15. 12. 1972 (IfZ, NL Schauff, Bd. 35).

47 Korrespondenz vom 10.9. und 20. 10. 1942 (IfZ, NL Schauff I, Bd. 8).

48 Korrespondenz Hermann von Freeden (im Auftrage Schauffs) mit dem Institut für Zeitgeschichte (Bibliothek/Dr.Vogelsang), 13.8. und 2.9. 1958 betr. Zusammenstellung von Literatur zur Emigration, die der Bibliotheksleiter um so lieber übermittelte, „als das Institut an Ihren und Herrn Dr. Schauffs Bemühungen sehr interessiert ist" (IfZ, NL Schauff, Bd. 39).

49 Vgl. Röder, Dokumentation, S. $54 \mathrm{ff}$. 
waren - wie Schauff - Teil eines nonkonformistischen informellen Netzwerks geworden. Der Erfahrungshintergrund solcher Persönlichkeiten, aber auch die vergleichsweise rasche Erschließung ihrer Papiere - privates Schriftgut und Akten von Parteien, Verbänden und Zirkeln, die sie geleitet oder in denen sie mitgewirkt hatten - bildeten im Institut für Zeitgeschichte eine wichtige Grundlage für die Erforschung des deutschsprachigen Exils ${ }^{50}$.

In diesem Rahmen war Schauff vor allem darum bemüht, auch jenen Teil des deutschsprachigen Exils in die Forschungen einzubeziehen, der nicht der Arbeiterbewegung zuzurechnen ist ${ }^{51}$; gleiches gilt für die Arbeit der Kommission für Zeitgeschichte ${ }^{52}$. Schauffs besonderes Interesse galt darüber hinaus dem inhaltlichen und organisatorischen Zusammenhang von Widerstand und Emigration. So versuchte er schon 1964 ein Forschungsprojekt zum „Widerstand jenseits der Reichsgrenzen" anzustoßen und führte in diesem Zusammenhang eine umfangreiche Korrespondenz mit dem ehemaligen saarländischen Ministerpräsidenten Johannes Hoffmann sowie dem Institut für Zeitgeschichte (Hans Buchheim) und der Kommission für Zeitgeschichte (Rudolf Morsey, Konrad Repgen) ${ }^{53}$.

Schauff war auch an den Bemühungen zur Erforschung von Widerstand und Emigration beteiligt, die von George N. Shuster, einem katholischen amerikanischen Universitätsprofessor und Vertrauten Brünings, ausgingen ${ }^{54}$. Er nahm an der Exiltagung teil, die vom 17. bis 19. Januar 1968 in Luxemburg stattfand und auf der sich - vor dem Hintergrund einer von der Deutschen Bibliothek organisierten Ausstellung zur „Exil-Literatur 1933-1945“ - Schriftsteller, Wissenschaft-

50 Vgl. Röder/Weiß/Lankheit, Das Archiv, S. $120 \mathrm{f}$.

51 Vgl. Schauffs Korrespondenz mit den damaligen Direktoren des IfZ, Helmut Krausnick bzw. Martin Broszat, sowie mit Horst Möller (damals Stellvertretender Direktor), ferner 1963-1980 mit Hans und Karl Buchheim, die auch Fragen der Erforschung der Geschichte Südtirols unter dem NS und des italienischen Faschismus betreffen (IfZ, NL Schauff, Bd. 1, 4, 5, 24); später fanden zahlreiche Gespräche mit dem Verfasser statt, der auch den Bereich des christlichen und konservativen Exils im Rahmen dieses biographischen Forschungsprojekts bearbeitete. Bei der öffentlichen Präsentation des „Biographischen Handbuchs der deutschsprachigen Emigration nach $1933^{\circ}$ am 9.7.1980 im Institut für Zeitgeschichte fand eine Podiumsdiskussion mit Johannes Schauff, Fritz Heine und Herbert Weichmann statt. Über dieses Podiumsgespräch, an dem auch die Historiker Morsey und Albrecht teilnahmen, schrieb Schauff an Gerhard Fittkau: „Ich habe mich zur Verfügung gestellt trotz erheblicher Bedenken, weil sonst die nicht-jüdische und nicht-sozialistische Emigration nicht vertreten gewesen wäre. Ich traf eine Menge Bekannte und bin mit dem Ergebnis zufrieden." (16. 7. 1980, IfZ, NL Schauff, Bd. 33).

52 Morsey an Schauff, 14. 12. 1982 (IfZ, NL Schauff, Bd. 5).

53 Weitere avisierte Mitarbeiter waren Walter Dirks und Georg Smolka. Ein erster konkreter Forschungsabschnitt, über dessen zu veröffentlichende Ergebnisse Schauff bereits mit dem Herder-Verlag verhandelte, war der Widerstand katholischer Publizisten 1933-1937 (u.a. Dietrich von Hildebrand, Friedrich Muckermann, Waldemar Gurian, Johannes Maier-Hultschin, Eduard Pant). Die betreffende Korrespondenz März bis Dezember 1964 in IfZ, NL Schauff, Bd. 39.

54 Vgl. die Korrespondenz Schauff-Shuster 1965 (IfZ, NL Schauff, Bd. 7); vgl. auch den Hinweis auf Shusters Bemühungen, Emigranten und Historiker zusammenzubringen, in: Heinrich Kronstein, Briefe, S. 319; zu Shusters Unterstützung der konservativen Emigration in den USA vgl. Schneider, Christliche und konservative Remigranten, S. $164 \mathrm{f}$. 
ler und Politiker trafen, die Deutschland nach 1933 aus politischen Gründen verlassen hatten 55 . Sie bildete den eigentlichen Anstoß für den Beginn der „Exilforschung" als einer eigenen zeitgeschichtlichen Disziplin.

55 Das Treffen fand unter dem Patronat des luxemburgischen Kultusministers Pierre Grégoire und des deutschen Außenministers Willy Brandt statt, der selbst auch anwesend war. Den Festvortrag zum Thema „Exil und Gegenwart" hielt Golo Mann; an einem Podiumsgespräch über „Nazismus, Widerstand, Exil und ihre Bedeutung für die Gegenwart“ waren beteiligt Hans Albert Kluthe, Richard Friedenthal, Alfred Frisch, Max Horkheimer, Hans Jaeger und Eva Reichmann (vgl. BHB I und II). Vgl. auch: 35 Jahre Exilliteratur, S. 135 ff.; IfZ, NL Schauff, Bd. 28 (Photodokumente). Das politische Exil war erstmals in der vorangegangenen Tagung des Forschungsinstituts der Friedrich-Ebert-Stiftung unter dem Titel "Widerstand, Verfolgung und Emigration 1933-1945" thematisiert worden, die vom 25.-30. 9. 1966 in der Heimvolkshochschule Bergneustadt stattfand (die Dokumentation dieser Tagung in der Reihe "Studien und Berichte aus dem Forschungsinstitut der Friedrich-Ebert-Stiftung “, Bad Godesberg 1967). 